\title{
A Review of Beneficial Low-Intensity Exercises in Diabetic Peripheral Neuropathy Patients
}

\author{
Carley Johnson ${ }^{1}$ and Jody K. Takemoto ${ }^{1,2}$ \\ ${ }^{1}$ Ben and Maytee Fisch College of Pharmacy and ${ }^{2}$ Department of Pharmaceutical Sciences, Ben and Maytee Fisch College \\ of Pharmacy, The University of Texas at Tyler
}

Received, August 27, 2018; Revised, December 11, 2018; Accepted, December 27, 2018; Published, December 27, 2018.

\begin{abstract}
The American Diabetic Association standards of medical care for diabetic patients recommends moderate intensity exercise to help manage diabetes; however, this recommendation may be unmanageable for patients who have become inactive or unable to reach this intensity. The purpose of this review is to determine if low-intensity exercises demonstrate improvement in diabetic peripheral neuropathy symptoms in order to utilize these exercises as a starting point for inactive patients. Studies in low-intensity exercises from 2013 to May 2018 were systematically searched in PubMed, SCOPUS, and Cochrane Library databases. The studies in this research have shown that low-intensity resistance exercises have promising outcomes such as improvements in pain interference with daily activities, pain thresholds, and reductions in neuropathy symptoms. Low-intensity aerobic therapy adds to the quality of life of the patient, and increases in strength of the lower limbs show an improvement in foot sensation and a reduction in pain and tingling symptoms.
\end{abstract}

\section{INTRODUCTION}

Diabetic peripheral neuropathy (DPN) is a complication of both type 1 and type 2 diabetes [1]. DPN has a complex pathogenesis marked by both metabolic and vascular factors [2]. Even though diabetes is a precursor to neuropathy, hyperglycemia is only one of the many key metabolic events known to cause axonal and microvascular injury [2]. It has been suggested that damage to small nerve fibers may precede damage to large nerve fibers which convey proprioception, innervate skeletal muscles, and mediate tendon reflexes [1]. Metabolic syndrome and obesity have also been linked to DPN $[3,4]$. In order to better manage diabetes, metabolic syndrome, and obesity, the recommendation for diabetic patients based on the ADA Guidelines is 150 minutes per week of moderate intensity exercise [5]. For patients who have become inactive or unable to reach that intensity, this recommendation may be unmanageable. Symptoms that become uncontrolled can lead to a decreased quality of life, increased risk of amputaiton, and increased medical costs [6]. Therefore, by incoporating low-intensity excerises into intial therapy, small increases in both the vascular and metabolic pathways could have potential to decrease painful neuropathic symptoms.

The purpose of this review is to determine if lowintensity exercises demonstrate improvement in DPN symptoms for pharmacists to utilize these exercises as a starting point for counseling in inactive patients. Although there are several studies on treatment modalities used to manage symptoms of DPN, nonpharmacological approaches such as lowintensity exercises have limited research. This review compares the outcomes of different types of low-intensity exercises studied within the last five years.

\section{MATERIALS AND METHODS}

We systematically searched PubMed, SCOPUS, and Cochrane Library databases for studies on exercise therapy from 2013 to December 2017. We narrowed the search of "exercise therapy" AND "peripheral neuropathy" to a more focused "low-intensity exercise" AND "peripheral neuropathy" within the realm of diabetes. Other searches used to find clinical trials and literature reviews include: "aerobic exercise" AND "peripheral neuropathy" and "yoga" AND "peripheral neuropathy." In looking at what to consider low-intensity, we followed the Centers for Disease Control and the U.S. Department of Health and Human Services physical activity guidelines (Figure 1).

Corresponding Author: Jody K. Takemoto, The University of Texas at Tyler, 3900 University Boulevard, Tyler, Texas 75799, Email: jtakemoto@uttyler.edu 


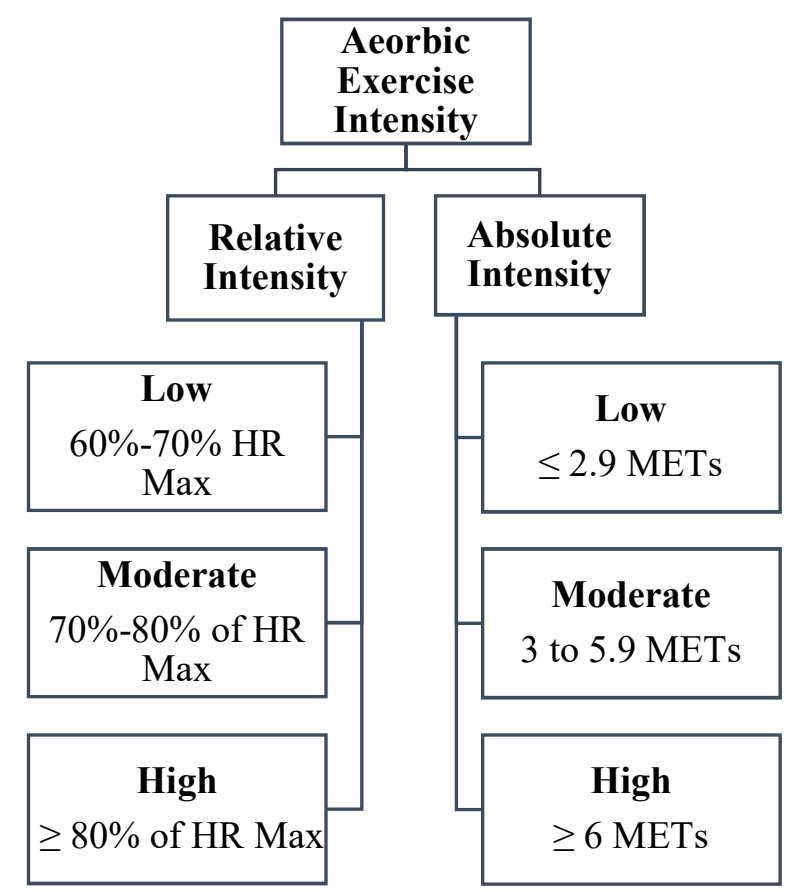

Figure 1. Categorizing Exercise Intensities. HR Max refers to the maximum heart rate of patient. MET refers to metabolic equivalent tasks This information is adapted from the Centers for Disease Control, the Office of Disease Prevention and Health Promotion [7,8].

We looked at these studies to see what benefit, if any, low-intensity weight bearing and non-weight bearing exercises have on DPN patients.

\section{RESULTS}

As seen in Table 1, a variety of low-intensity exercise techniques have been studied in evaluating benefits in diabetic neuropathy patients. When categorizing which exercises are considered aerobic, resistance, balance, or a combination, we followed the U.S. Department of Health and Human Services physical activity guidelines [8]. The exercises are categorized by the focus of each study. Aerobic exercises are walking, mini-trampoline exercises, and aquatic therapy. Resistance exercises are those completed using additional weights or weight machines with the intention of building muscle. Balance exercises are exercises solely targeted at improving balance. Combination exercises are compound movements that incorporate multiple exercises such as tai chi and yoga.

\section{Aerobic exercise}

When determining what qualifies as aerobic exercise, the U.S. Department of Health and Human Services physical activity guidelines is the starting point [8]. Classifying exercises such as walking, mini-trampoline, and aquatic exercises as aerobic was based on the guidelines (Figure 1) and determined either by the studies mentioning lowintensity.

There were two studies that showed improvements with walking. Yoo et al. was a pilot study looking at exercise in previously inactive diabetic patients $(n=14)$ with peripheral neuropathy [9]. This study focused on implementing normal walking three times a week for 30 minutes increasingly adding time to 50 minutes over a period of 16 weeks. Participants were between the ages of 40 and 70 with diabetes and symptoms of peripheral neuropathy. Pain was assessed using the Brief Pain Inventory Short Form for Diabetic Peripheral Neuropathy. This scale has a pain intensity component as well as pain interference assessments [9]. Although the data did not suggest a reduction in pain intensities $(p=0.34)$, the results showed a statistically significant improvement in pain interference with daily activities such as walking $(\mathrm{p}=0.016)$, sleep $(\mathrm{p}=0.02)$, and relationships with others $\quad(p=0.006)$ Additionally, participants improved their threshold for intensity of aerobic exercise $(p=0.028)$. In another study, mentioned under the title "resistance exercise" heading below, 
there were also improvements seen with lowintensity walking.

In addition to walking, a low-intensity minitrampoline exercise was considered. This study was a controlled clinical trial comparing patients using both weight and non-weight-bearing exercises on a mini-trampoline. Participants in the study was narrowed to a population of 24. Outcome measures were foot mobility (right foot, $\mathrm{p}=0.40$; left foot, $\mathrm{p}=0.012$ ), plantar pressure (right foot, $\mathrm{p}=0.016$; left foot, $\mathrm{p}=0.034)$, and sensation $(\mathrm{p}=0.008-0.031)$ of feet in diabetic neuropathy patients $(n=12)$ [10]. Participants completed trampoline exercises five times a week for a total of an 8-week study (every two weeks the level of difficulty increased). The study found that the mini-trampoline exercises reduced the risk of foot ulcers while also increasing muscle strength and balance. The outcomes of this study were measured using range of motion technique and Footscan 7 hardware. The two peripheral neuropathy outcomes were measured utilizing the 10-g monofilament test and neuropathy and foot-ulcer-specific quality of life instrument.

Lastly, there was one comparative study of aquatic versus land therapy in low-intensity aerobic exercise. This was a parallel-group, single-center, single-blind randomized control trial. The intervention compared the same therapy in water $(n=21)$ with the same therapy on land $(n=19)$ with the measurement outcomes of gait and balance in patients with peripheral neuropathy $(\mathrm{n}=40)$ [11]. The study showed improvement in gait with aquatic therapy in comparison to land therapy (control group); Dynamic Gait Index $(\mathrm{p}=0.04)$ and Berg Balance Scale $(p=0.86)$ were the outcome measurements in water therapy while non-aquatic therapy utilized the Berg Balance Scale $(\mathrm{p}=0.024)$.

Table 1. Low-Intensity Exercise Benefits

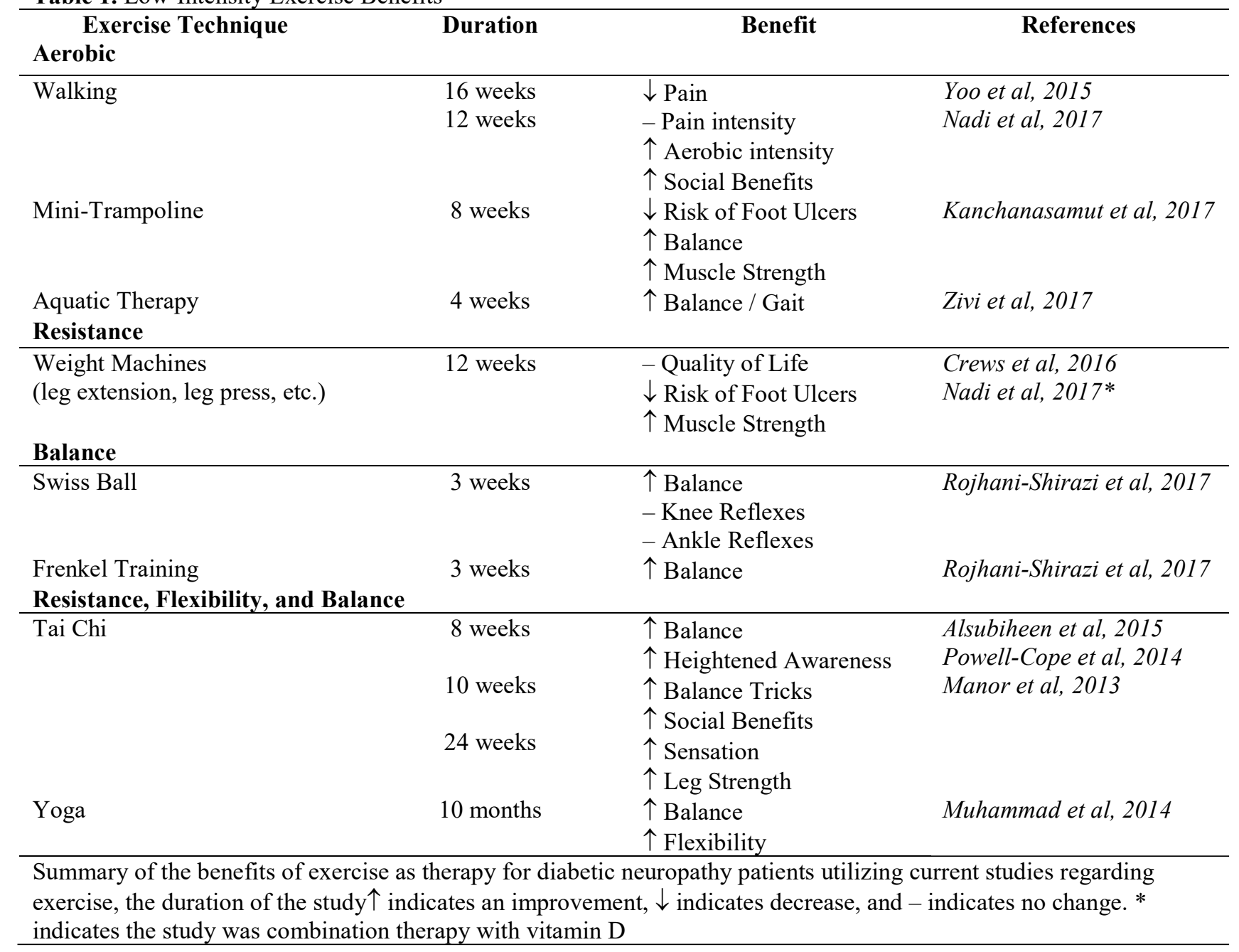


Aquatic therapy showed no change in the impairment of everyday activities or quality of life experienced $(p=0.27)$, and neuropathic pain did not improve with land therapy $(\mathrm{p}=0.46)$.

\section{Resistance exercise}

When determining what qualifies as resistance exercise, the U.S. Department of Health and Human Services physical activity guidelines was utilized [8]. Additionally, the only resistance exercise study was in combination with aerobic exercise. The aerobic intensity was evaluated using Figure 1 while the resistance exercises were determined by the Michigan Neuropathy Screening Instrument, reflex hammer, and tuning fork. These measurements are used to assess distal symmetrical peripheral neuropathy in diabetes [12].

The most recent study in resistance exercise is the combination study of aerobic exercise and resistance training with vitamin $\mathrm{D}$ therapy in female patients with diabetic neuropathy $(n=90)$ [13]. This exercise training consisted of 60 minutes of training 3 days a week for 12 weeks. The combined training and vitamin $\mathrm{D}$ therapy showed a reduction in tingling $(\mathrm{p}=0.001)$, numbness $(\mathrm{p}=0.001)$, pain $(\mathrm{p}=0.002)$, and weakness in the lower limbs $(p=0.002)$ but no significant change in knee $(p=0.77)$ or ankle $(p=0.47)$ reflexes. The outcomes of this study were measured using the pre-post interventions with commonly used measures, specifically, the Michigan neuropathy questionnaire, reflex hammer, and tuning fork tests.

\section{Balance exercise}

The U.S. Department of Health and Human Services physical activity guidelines was used to determine what qualified as balance exercise [8]. RojahaniShirazi et al. measured balance in patients with neuropathy by comparing three arms: a control group $(\mathrm{n}=20)$, ball training group $(\mathrm{n}=20)$, and Frenkel training group $(n=20)$ [14]. Frenkel exercises were divided into lying, sitting, and standing positions each requiring flexion, extension, abduction and adduction exercises [14]. Whereas, ball training requires the same active movements such as flexion, extension, abduction and adduction but while utilizing the Swiss ball. Exercise training for 55 minutes, 5 days a week for 3 weeks showed significant difference between both exercises in balance tests. The one leg standing tests observed a significant increase from control in both ball training $(p<0.001)$ and Frenkel $(p=0.01)$ training. Also, the
Berg Balance Test showed a significant increase in ball training $(\mathrm{p}<0.01)$ and Frenkel training $(\mathrm{p}=0.01)$. In addition to the Berg Balance Test, other outcomes were measured with the one leg stance test and the Star Excursion Balance Test.

\section{Resistance, flexibility, and balance combination exercise}

When determining what qualifies as a combination exercise, the U.S. Department of Health and Human Services physical activity guidelines is the starting point [8]. These studies have been studied in diabetes within the recent years. These studies define Tai Chi as having clinical measures of balance, musculoskeletal strength and flexibility which also helped to categorize these exercises in the combination category $[15,16]$.

Powell-Cope et al. studied Tai Chi - Yang Style $(n=17)$ and Functional Based Training (FBT) $(n=19)$ interventions in patients [15]. The study compared short term (6 weeks) and long term (6 months) outcomes of training 1 hour per week. These combination exercise therapies measured qualitative improvements in patients' perceptions of balance (FBT 6 weeks only), balance tricks, heightened awareness of walking (6 weeks only), and social benefits. Similarly, Manor et al. studied the effect of Tai Chi - Yang Style in patients with peripheral neuropathy $(\mathrm{n}=25)$, eight of which had diabetes [16]. Foot sole sensation $(\mathrm{p}=0.003)$, leg strength measured by knee extensor $(\mathrm{p}=0.009)$ and flexor peak torque $(\mathrm{p}=0.01)$, and physical function measured by six-minute walk test $(p<0.001)$ and TUG $(\mathrm{p}<0.001)$ all improved over a span of 24 weeks. Outcomes were measured using the centerof-pressure dynamics, 5.07 gauge SemmesWeinstein monofilament, Biodex dynamometer, and timed up-and-go test.

\section{DISCUSSION}

Low-intensity exercises do show improvements in diabetic patients experiencing neuropathy [9-18]. The outcomes of each study vary in respect to what is being measured. Some studies focused on alleviating pain or pain interference [9] while others focused on preventing further complications such as falling, ulcers, or amputations [10, 13-18]. Because the exercises have different intentions, making a comparison to rank which exercise is most beneficial for patients with neuropathy is not possible. Instead, a more appropriate way to apply these studies 
suggest exercises should be tailored to subpopulations with diabetic peripheral neuropathy based on their outcome measurement scales.

Aerobic exercises, alone, at a lower intensity thus far does not show any benefit therapeutically to the diabetic patient in reference to neither $\mathrm{HbA} 1 \mathrm{C}$ and blood glucose nor pain intensities [9]; however, beginning with a low-intensity exercise improved quality of life [9]. More randomized controlled trials are needed to show benefit. In a patient with too high of a pain intensity to walk on land could experience an improvement in daily activities with aquatic therapy [11]. Additionally, another non-weight bearing exercise patients could use to see an improvement in balance is Swiss ball training [14]. Tai-Chi, a combination of resistance, flexibility, and balance exercises, showed more benefits in both quality of life and peripheral neuropathy symptoms [15-18]. Also, aerobic exercises in combination with resistance training with vitamin $\mathrm{D}$ showed improvement in peripheral neuropathy symptoms [13].

Collectively data suggests that resistance therapy may be essential in improving neuropathic pain. Low-intensity aerobic therapy adds to the quality of life of the patient but strengthening lower limbs show a clinical improvement in foot sensation as well as a reduction of pain and tingling symptoms. Therefore, by improving the clinical symptoms of peripheral neuropathy with low-intensity exercises, patients may then become able to move forward to the American Diabetic Association recommendation of moderate intensity exercises to ultimately better manage both the diabetes and pain.

Understanding, preventing, and managing diabetic peripheral neuropathy and its complications is imperative for improving patients' quality of life. Although only a limited number of studies on lowintensity exercise therapy have been completed in recent years, each show improvements in patient outcomes including quality of life, decreased pain intensities, and increased balance. However, when comparing the different studies, there are no set guidelines, requirements, or measurement outcome tools utilized in DPN for every study. Because of the varieties in the types of outcome tools, the data cannot be fairly compared. The studies in this research have shown that low-intensity resistance exercises have promising outcomes where pharmacists can advocate for patient's quality of life. As research in DPN develops, more trials in exercises should be completed. Specifically, combination of exercise and medication therapies, comparisons in length and duration of exercises, and studies in types of exercises could contribute noteworthy evidence for DPN patients striving to manage their symptoms. One underlying issue with each of these studies is the lack of a gold standard in measuring outcomes to determine any quantitative or qualitative improvements; therefore, integrating a system of universal measurements in DPN is imperative. This research is novel as this is the first report to compare low-intensity exercises showing benefits in DPN patients in which pharmacists can use to provide non-pharmacological counseling.

\section{ACKNOWLEDGMENTS}

The authors would like to thank Dr. Takova Wallace, Pharm D, BCPS from the Ben and Maytee Fisch College of Pharmacy at the University of Texas at Tyler for her advice in developing the foundation of this research.

\section{REFERENCES}

1. Javed, S., Alam, U. and Malik, R.A. 2015. Treating diabetic neuropathy: present strategies and emerging solutions. The review of diabetic studies : RDS 12(12), pp. 63-83.

2. Juster-Switlyk, K. and Smith, A.G. 2016. Updates in diabetic peripheral neuropathy. [version 1; referees: 3 approved]. F1000Research 5.

3. Singleton, J.R., Marcus, R.L., Jackson, J.E., K Lessard, M., Graham, T.E. and Smith, A.G. 2014. Exercise increases cutaneous nerve density in diabetic patients without neuropathy. Annals of clinical and translational neurology 1(10), pp. 844849.

4. Singleton, J.R., Marcus, R.L., Lessard, M.K., Jackson, J.E. and Smith, A.G. 2015. Supervised exercise improves cutaneous reinnervation capacity in metabolic syndrome patients. Annals of Neurology 77(1), pp. 146-153.

5. American Diabetes Association 2018. 4. Lifestyle Management: Standards of Medical Care in Diabetes2018. Diabetes Care 41(Suppl 1), pp. S38-S50.

6. Edwards, J.L., Vincent, A.M., Cheng, H.T. and Feldman, E.L. 2008. Diabetic neuropathy: mechanisms to management. Pharmacology \& Therapeutics 120(1), pp. 1-34.

7. President's Council on Sports, Fitness \& Nutrition. (2017, January) Physical Activity Guidelines for Americans. Retrieved from https://www.hhs.gov/fitness/be-active/physicalactivty-guidelines-for-americans/index.html. 
8. Office of Disease Prevention and Health Promotion. (2018, May) Chapter 4: Active Adults. Retrieved from

https://health.gov/paguidelines/guidelines/chapter4.a spx

9. Yoo, M., D’Silva, L.J., Martin, K., Sharma, N.K., Pasnoor, M., LeMaster, J.W. and Kluding, P.M. 2015. Pilot study of exercise therapy on painful diabetic peripheral neuropathy. Pain Medicine 16(8), pp. 1482-1489.

10. Kanchanasamut, W. and Pensri, P. 2017. Effects of weight-bearing exercise on a mini-trampoline on foot mobility, plantar pressure and sensation of diabetic neuropathic feet; a preliminary study. Diabetic foot \& ankle 8(1), p. 1287239.

11. Zivi, I., Maffia, S., Ferrari, V., Zarucchi, A., Molatore, K., Maestri, R. and Frazzitta, G. 2017. Effectiveness of aquatic versus land physiotherapy in the treatment of peripheral neuropathies: a randomized controlled trial. Clinical Rehabilitation, p. 269215517746716.

12. Herman, W.H., Pop-Busui, R., Braffett, B.H., Martin, C.L., Cleary, P.A., Albers, J.W., Feldman, E.L. and DCCT/EDIC Research Group 2012. Use of the Michigan Neuropathy Screening Instrument as a measure of distal symmetrical peripheral neuropathy in Type 1 diabetes: results from the Diabetes Control and Complications Trial/Epidemiology of Diabetes Interventions and Complications. Diabetic Medicine 29(7), pp. 937-944.

13. Nadi, M., Marandi, S.M., Esfarjani, F., Saleki, M. and Mohammadi, M. 2017. The Comparison between
Effects of 12 weeks Combined Training and Vitamin D Supplement on Improvement of Sensory-motor Neuropathy in type 2 Diabetic Women. Advanced biomedical research 6, p. 55.

14. Rojhani-Shirzi, Z., Barzintaj, F., and Salimifard, M. Comparison the effects of two types of therapeutic exercises vs. Swiss ball on the clinical balance measures in patients with diabetic neuropathy. Diabetes and Metabolic Syndromes: Clinical Research and Reviews. 11S pp S29-S32.

15. Powell-Cope, G., Quigley, P.A., Besterman-Dahan, K. and Lind, J.D. 2014. Perceived benefits of group exercise among individuals with peripheral neuropathy. Western journal of nursing research 36(7), pp. 855-874.

16. Manor, B., Lipsitz, L.A., Wayne, P.M., Peng, C.K. and Li, L. 2013. Complexity-based measures inform Tai Chi's impact on standing postural control in older adults with peripheral neuropathy. $B M C$ complementary and alternative medicine 13, p. 87.

17. Crews, R.T., Schneider, K.L., Yalla, S.V., Reeves, N.D. and Vileikyte, L. 2016. Physiological and psychological challenges of increasing physical activity and exercise in patients at risk of diabetic foot ulcers: a critical review. Diabetes/Metabolism Research and Reviews 32(8), pp. 791-804.

18. Alsubiheen, A., Petrofsky, J., Daher, N., Lohman, E. and Balbas, E. 2015. Effect of Tai Chi Exercise Combined with Mental Imagery Theory in Improving Balance in a Diabetic and Elderly Population. Medical Science Monitor 21, pp. 3054-3061. 